\title{
Using Differential Transform Method and Padé Approximant for Solving MHD Flow in a Laminar Liquid Film from a Horizontal Stretching Surface
}

\author{
Mohammad Mehdi Rashidi and Mohammad Keimanesh \\ Mechanical Engineering Department, Engineering Faculty of Bu-Ali Sina University, \\ P.O. Box 65175-4161 Hamedan, Iran \\ Correspondence should be addressed to Mohammad Mehdi Rashidi,mm_rashidi@yahoo.com
}

Received 12 November 2009; Accepted 20 January 2010

Academic Editor: Mehrdad Massoudi

Copyright (c) 2010 M. M. Rashidi and M. Keimanesh. This is an open access article distributed under the Creative Commons Attribution License, which permits unrestricted use, distribution, and reproduction in any medium, provided the original work is properly cited.

\begin{abstract}
The purpose of this study is to approximate the stream function and temperature distribution of the MHD flow in a laminar liquid film from a horizontal stretching surface. In this paper DTM-Padé method was used which is a combination of differential transform method (DTM) and Padé approximant. The DTM solutions are only valid for small values of independent variables. Comparison between the solutions obtained by the DTM and the DTM-Pade with numerical solution (fourth-order Runge-Kutta) revealed that the DTM-Padé method is an excellent method for solving MHD boundary-layer equations.
\end{abstract}

\section{Introduction}

As the researches indicated, the nonlinear equations are one of the most important phenomena across the world. Nonlinear phenomena have important effects on applied mathematics, physics, and issues related to engineering. Then the variation of each parameter depends on different factors. The importance of obtaining the exact or approximate solutions of nonlinear partial differential equations (NLPDEs) in physics and mathematics is still a big problem that needs new methods to discover new exact or approximate solutions. Most of nonlinear equations do not have a precise analytic solution; so numerical methods have largely been used to handle these equations. There are also some analytic techniques for nonlinear equations. Some of the classic analytic methods are Lyapunov's artificial small parameter method [1], perturbation techniques [2-11], and $\delta$-expansion method [12]. In the recent years, many authors mainly had paid attention to study solutions of nonlinear partial differential equations by using various methods. Among these are the Adomian decomposition method (ADM) [13, 14], tanh method, homotopy perturbation method (HPM), sinh-cosh method, HAM, the DTM, and variational iteration method (VIM) $[15,16]$. 
Magnetohydrodynamics (MHD) is the study of the interaction of conducting fluids with electromagnetic phenomena. The flow of an electrically conducting fluid in the presence of a magnetic field is of importance in various areas of technology and engineering such as MHD power generation, MHD flow meters, and MHD pumps [17-22]. The viscous flow due to stretching boundary is important in extrusion processes where sheet material is pulled out of an orifice with increasing velocity. If the boundary velocity is linear with respect to a fixed point, exact solutions of the Navier-Stokes equations may be obtained [23, 24].

In recent years the analysis of fluid flow across a thin liquid film has attracted the attention of a number of researchers because of its possible applications in many branches of science and technology. The knowledge of flow and heat transfer within a thin liquid film is crucial in understanding the coating process and design of various heat exchangers and chemical processing equipments. Other applications include wire and fiber coating, food stuff processing, reactor fluidization, and transpiration cooling. The prime aim in almost every extrusion applications is to maintain the surface quality of the extrudate. All coating processes demand a smooth glossy surface to meet the requirements for best appearance and optimum service properties such as low friction, transparency, and strength. The problem of extrusion of thin surface layers needs special attention to gain some knowledge for controlling the coating product efficiently.

The motivation of this letter is the use of the differential transform method and Padé approximant to construct analytical approximate solutions of the Heat transfer in a liquid film over an unsteady stretching surface with viscous dissipation in presence of external magnetic field. The concept of differential transform method was first introduced by Zhou [25] in 1986 and it was used to solve both linear and nonlinear initial value problems in electric circuit analysis. The main advantage of this method is that it can be applied directly to NLPDEs without requiring linearization, discretization, or perturbation. It is a semianalytical-numerical technique that formulizes Taylor series in a very different manner. This method constructs, for differential equations, an analytical solution in the form of a polynomial. Not like the traditional high-order Taylor series method that requires symbolic computation, the DTM is an iterative procedure for obtaining Taylor series solutions. Another important advantage is that this method reduces the size of computational work while the Taylor series method is computationally taken long time for large orders. This method is well addressed in [26-33].

\section{Mathematical Formulation}

\subsection{Governing Equations and Boundary Conditions}

Consider a thin elastic liquid film of uniform thickness $h(t)$ lying on the horizontal stretching sheet. The $x$-axis is chosen in the direction along which the sheet is set to motion and the $y$-axis is taken perpendicular to it. The fluid motion within the film is primarily caused solely by stretching of the sheet. The sheet is stretched by the action of two equal and opposite forces along the $x$-axis. The sheet is assumed to have velocity $U$ and the flow field is exposed to the influence of an external transverse magnetic field of strength $B$. We have neglected the effect of latent heat due to evaporation by assuming the liquid to be nonvolatile. Further the buoyancy is neglected due to the relatively thin liquid film, but it is not so thin that intermolecular forces come into play. The velocity and temperature fields of the liquid film obey the following boundary-layer equations: 


$$
\begin{gathered}
\frac{\partial u}{\partial x}+\frac{\partial v}{\partial y}=0 \\
\frac{\partial u}{\partial t}+u \frac{\partial u}{\partial x}+v \frac{\partial u}{\partial y}=v \frac{\partial^{2} u}{\partial y^{2}}-\frac{\sigma B^{2}}{\rho} u \\
\frac{\partial T}{\partial t}+u \frac{\partial T}{\partial x}+v \frac{\partial T}{\partial y}=\frac{k}{\rho C_{p}} \frac{\partial^{2} T}{\partial y^{2}}+\frac{\mu}{\rho C_{p}}\left(\frac{\partial u}{\partial y}\right)^{2}
\end{gathered}
$$

The pressure in the surrounding gas phase is assumed to be uniform and the gravity force gives rise to a hydrostatic pressure variation in the liquid film. In order to justify the boundary-layer approximation, the length scale in the primary flow direction must be significantly larger than the length scale in the cross stream direction. We choose the representative measure of the film thickness to be $(v / b)^{1 / 2}$ so that the scale ratio is large enough. This choice of length scale enables us to employ the boundary-layer approximations. Further it is assumed that the induced magnetic field is negligibly small. The associated boundary conditions are given by

$$
\begin{gathered}
u=U, \quad v=0, \quad T=T_{s} \quad \text { at } y=0, \\
\frac{\partial u}{\partial y}=\frac{\partial T}{\partial y}=0 \quad \text { at } y=h, \\
v=\frac{d h}{d t} \quad \text { at } y=h .
\end{gathered}
$$

At this juncture we make a note that the mathematical problem is implicitly formulated only for $x \geq 0$. Further it is assumed that the surface of the planar liquid film is smooth so as to avoid the complications due to surface waves. The influence of interfacial shear due to the quiescent atmosphere, in other words the effect of surface tension, is assumed to be negligible. The viscous shear stress $\tau=\mu(\partial u / \partial y)$ and the heat flux $q=-k(\partial T / \partial y)$ vanish at the adiabatic free surface (at $y=h$ ).

Let us consider a thin elastic sheet which emerges from a narrow slit at the origin of a Cartesian coordinate system. The continuous sheet at $y=0$ is parallel with the $x$-axis and moves in its own plane with the velocity

$$
U(x, t)=\frac{b x}{1-\alpha t}
$$

where $b$ and $\alpha$ are both positive constants with dimension per time. The surface temperature $T_{S}$ of the stretching sheet is assumed to vary with the distance $x$ from the slit as

$$
T_{s}(x, t)=T_{0}-T_{\text {ref }}\left[\frac{b x^{2}}{2 v}\right]
$$

where $T_{0}$ is the temperature at the slit and $T_{\text {ref }}$ can be taken as a constant reference temperature such that $0 \leq T_{\text {ref }} \leq T_{0}$. The term $b x^{2} / v(1-\alpha t)$ can be recognized as the local Reynolds 
number based on the surface velocity $U$. The expression (2.7) for the velocity of the sheet $U(x, t)$ reflects that the elastic sheet which is fixed at the origin is stretched by applying a force in the positive $x$-direction and the effective stretching rate $b /(1-\alpha t)$ increases with time as $0 \leq \alpha<1$. With the same analogy the expression for the surface temperature $T_{s}(x, t)$ given by (2.8) represents a situation in which the sheet temperature decreases from $T_{0}$ at the slit in proportion to $x^{2}$ and such that the amount of temperature reduction along the sheet increases with time. The applied transverse magnetic field is assumed to be of variable kind and is chosen in its special form as

$$
B(x, t)=B_{0}(1-\alpha t)^{-1 / 2} .
$$

The particular form of the expressions for $U(x, t), T_{s}(x, t)$, and $B(x, t)$ are chosen so as to facilitate the construction of a new similarity transformation which enables in transforming the governing partial differential equations of momentum and heat transfer into a set of nonlinear ordinary differential equations.

\subsection{Similarity Transformations}

We now introduce dimensionless variables $f$ and $q$ and the similarity variable $\eta$ as

$$
\begin{gathered}
f(\eta)=\frac{\psi(x, y, t)}{(v b /(1-\alpha t))^{x}} \\
\theta(\eta)=\frac{T_{0}-T(x, y, t)}{T_{\text {ref }}\left(b x^{2} / 2 v(1-\alpha \mathrm{t})^{-3 / 2}\right)^{2}} \\
\eta=\left(\frac{b}{v(1-\alpha t)}\right)^{1 / 2} .
\end{gathered}
$$

The physical stream function $\psi(x, y, t)$ automatically assures mass conversion given in (2.1). The velocity components are readily obtained as

$$
\begin{gathered}
u=\frac{\partial \psi}{\partial y}\left(\frac{b x}{1-\alpha t}\right) f^{\prime}(\eta), \\
v=-\frac{\partial \psi}{\partial y}=-\left(\frac{v b}{1-\alpha t}\right)^{1 / 2} f(\eta) .
\end{gathered}
$$

The system of partial differential equations (2.1)-(2.3) with boundary conditions (2.4)-(2.6) transforms exactly into a set of ordinary differential equations and their associated boundary 
conditions [34]:

$$
\begin{gathered}
S\left(f^{\prime}+\frac{\eta}{2} f^{\prime \prime}\right)+\left(f^{\prime}\right)^{2}-f f^{\prime \prime}=f^{\prime \prime \prime}-M n f^{\prime}, \\
\operatorname{Pr}\left[\frac{S}{2}\left(3 \theta+\eta \theta^{\prime}\right)+2 \theta f^{\prime}-\theta^{\prime} f\right]=\theta^{\prime \prime}+E c \operatorname{Pr} f^{\prime \prime 2}, \\
f^{\prime}(0)=1, \quad f(0)=0, \quad \theta(0)=1, \\
f^{\prime \prime}(\beta)=0, \quad \theta^{\prime}(\beta)=0, \\
f(\beta)=\frac{S \beta}{2} .
\end{gathered}
$$

Here $S \equiv \alpha / b$ is the dimensionless measure of the unsteadiness and the prime indicates differentiation with respect to $\eta$. Further, $\beta$ denotes the value of the similarity variable $h$ at the free surface so that (2.12) gives

$$
\beta=\left(\frac{b}{v(1-\alpha t)}\right)^{1 / 2} h
$$

Yet $b$ is an unknown constant, which should be determined as an integral part of the boundary value problem. The rate at which film thickness varies can be obtained differentiating equation (2.20) with respect to $t$, in the form

$$
\frac{d h}{d t}=-\frac{\alpha \beta}{2}\left(\frac{v}{b(1-\alpha t)}\right)^{1 / 2}
$$

Thus the kinematics constraint at $y=h(t)$ given by (2.6) transforms into the free surface condition (2.21). It is noteworthy that the momentum boundary-layer equation defined by (2.15) subject to the relevant boundary conditions (2.17)-(2.19) is decoupled from the thermal field; on the other hand the temperature field $\theta(\eta)$ is coupled with the velocity field $f(\eta)$. Since the sheet is stretched horizontally, the convection least affects the flow (i.e., buoyancy effect is negligibly small) and hence there is a one-way coupling of velocity and thermal fields.

The local skin friction coefficient, which of practical importance, is given by

$$
C_{f} \equiv \frac{-2 \mu(\partial u / \partial y)_{y=0}}{\rho U^{2}}=-2 \operatorname{Re}_{x}{ }^{-1 / 2} f^{\prime \prime}(0)
$$

and the heat transfer between the surface and the fluid conventionally expressed in dimensionless form as a local Nusselt number is given by

$$
N u_{x} \equiv-\frac{x}{T_{\text {ref }}}\left(\frac{\partial T}{\partial y}\right)_{y=0}=\frac{1}{2}(1-\alpha t)^{-1 / 2} \theta^{\prime}(0) \operatorname{Re}_{x}^{3 / 2},
$$


Table 1

\begin{tabular}{ll}
\hline Original function & Transformed function \\
\hline$f(t)=u(t) \pm v(t)$ & $F(k)=U(k) \pm V(k)$ \\
$f(t)=\lambda u(t)$ & $F(k)=\frac{(k+n) !}{k !} U(k+n)$ \\
$f(t)=\frac{d^{n} u(t)}{d t^{n}}$ & $\left.F(k)=\sum_{r=0}^{k} \delta(r-1)(k-r+1) U(k-r+1)\right)$ \\
$f(t)=t \frac{d u(t)}{d t}$ & $F(k)=\sum_{r=0}^{k} \delta(r-1)(k-r+1)(k-r+2) U(k-r+2)$ \\
$f(t)=t \frac{d^{2} u(t)}{d t^{2}}$ & $F(k)=\sum_{r=0}^{k}(r+1)(k-r+1) U(r+1) U(k-r+1)$ \\
$f(t)=\frac{d u(t)}{d t} \frac{d u(t)}{d t}$ & $F(k)=\sum_{r=0}^{k}(k+1)(r+2)(k-r+2)$ \\
$f(t)=\frac{d^{2} u(t)}{d t^{2}} \frac{d^{2} u(t)}{d t^{2}}$ & $F(k)=\sum_{r=0}^{k}(k-r+2)(k-r+1) U(r) U(k-r+2)$ \\
$f(t)=u(t) \frac{d^{2} u(t)}{d t^{2}}$ & \\
\hline
\end{tabular}

where $\operatorname{Re}_{x}=U x / v$ denotes the local Reynolds number and $T_{\text {ref }}$ denotes the same reference temperature (temperature difference) as in (2.8). We now march on to find the solution of the boundary value problem (2.15)-(2.19).

\section{The Differential Transform Method}

Transformation of the $k$ th derivative of a function in one variable is as follows [35]:

$$
F(k)=\frac{1}{k !}\left[\frac{d^{k} f(t)}{d t^{k}}\right]_{t=t_{0}},
$$

and the inverse transformation is defined by [36]

$$
\begin{aligned}
& f(t)=\sum_{k=0}^{\infty} F(k)\left(t-t_{0}\right)^{k}, \\
& f(t) \cong \sum_{k=0}^{i} F(k)\left(t-t_{0}\right)^{k},
\end{aligned}
$$

where $F(k)$ is the differential transform of $f(t)$. 
Taking differential transform of (2.15)-(2.16) by using the related definitions in Table 1, we obtain

$$
\begin{aligned}
& S\left((k+1) F(k+1)+\frac{1}{2} \sum_{r=0}^{k} \delta(r-1)(k-r+1)(k-r+2) F(k-r+2)\right) \\
& \quad+\sum_{r=0}^{k}(r+1)(k-r+1) F(r+1) F(k-r+1)-\sum_{r=0}^{k}(k-r+2)(k-r+1) F(r) F(k-r+2) \\
& \quad=(k+1)(k+2)(k+3) F(k+3)-M n(k+1) F(k+1), \\
& \operatorname{Pr}\left[\frac{S}{2}\left(3 \Theta(k)+\sum_{r=0}^{k} \delta(r-1)(k-r+1) \Theta(k-r+1)\right)\right. \\
& \left.+2 \sum_{r=0}^{k}(k-r+1) \Theta(r) F(k-r+1)-\sum_{r=0}^{k}(k-r+1) \Theta(k-r+1) F(r)\right] \\
& =(k+1)(k+2) \Theta(k+2)+E c \operatorname{Pr} \sum_{r=0}^{k}(r+1)(r+2)(k-r+2)(k-r+1)
\end{aligned}
$$

and by using the DTM, the boundary conditions ((2.17) and (2.18)) are transformed into a recurrence equation that finally leads to the solution of a system of algebraic equations. We can consider the boundary conditions (3.4) as follows:

$$
\begin{gathered}
f^{\prime}(0)=1, \quad f(0)=0, \quad \theta(0)=1, \\
f^{\prime \prime}(\beta)=0, \quad \theta^{\prime}(\beta)=0 .
\end{gathered}
$$

The differential transform of the boundary conditions is as follows:

$$
\begin{gathered}
F(1)=1, \quad F(0)=0, \quad \Theta(0)=1, \\
\sum_{k=0}^{\infty} k(k-1) \beta^{k-2} F(k)=0, \quad \sum_{k=0}^{\infty} k \beta^{k-1} \Theta(k)=0 .
\end{gathered}
$$

\section{Results and Discussion}

System of nonlinear ordinary differential equations (3.4) with transformed boundary conditions was solved analytically using the DTM and the DTM-Padé. As there are not exact solutions for nonlinear equations, a comparison of analytical and numerical solutions was conducted. In numerical solutions, the fourth-order Runge-Kutta method was applied. It should be noted that the solution exists only for small value of unsteadiness parameter $0 \leq S \leq 2$.

After finding the DTM solutions for (3.4), the Padé approximant must be applied. Ismail and Abd Rabboh [37] presented a restrictive Padé approximation for the generalized 


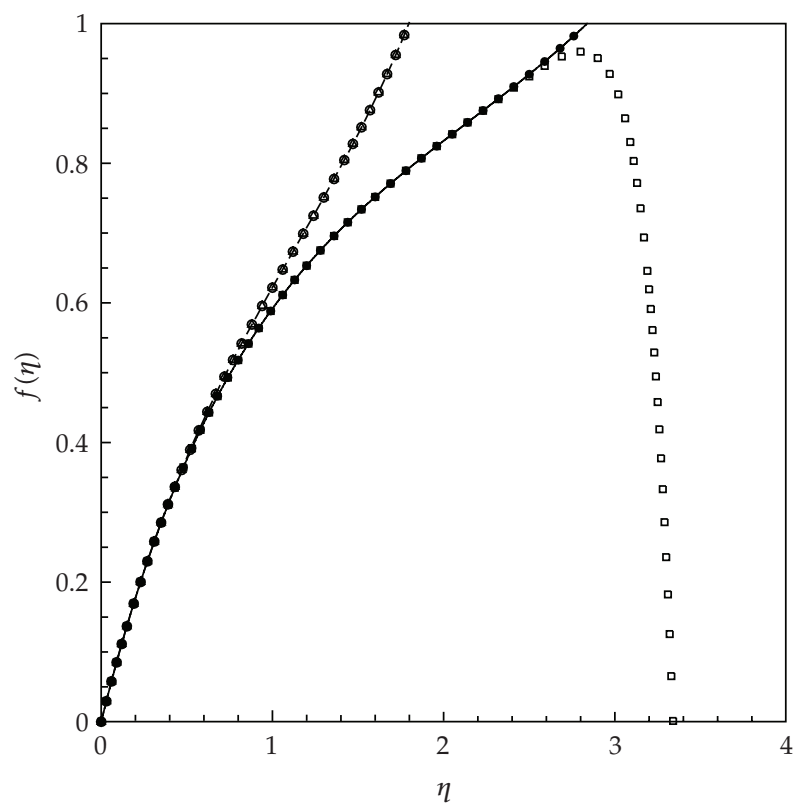

- $\quad$ Numerical, $S=0.8 \quad$ o Numerical, $S=1.2$

- $\quad \mathrm{DTM}, S=0.8 \quad \Delta \quad \mathrm{DTM}, S=1.2$

- DTM-Padé $[10,10], \quad$ - - DTM-Padé $[10,10]$,

Figure 1: Comparison of the solutions obtained by the DTM and the DTM-Pade with numerical solutions for $M n=0, E c=0$.

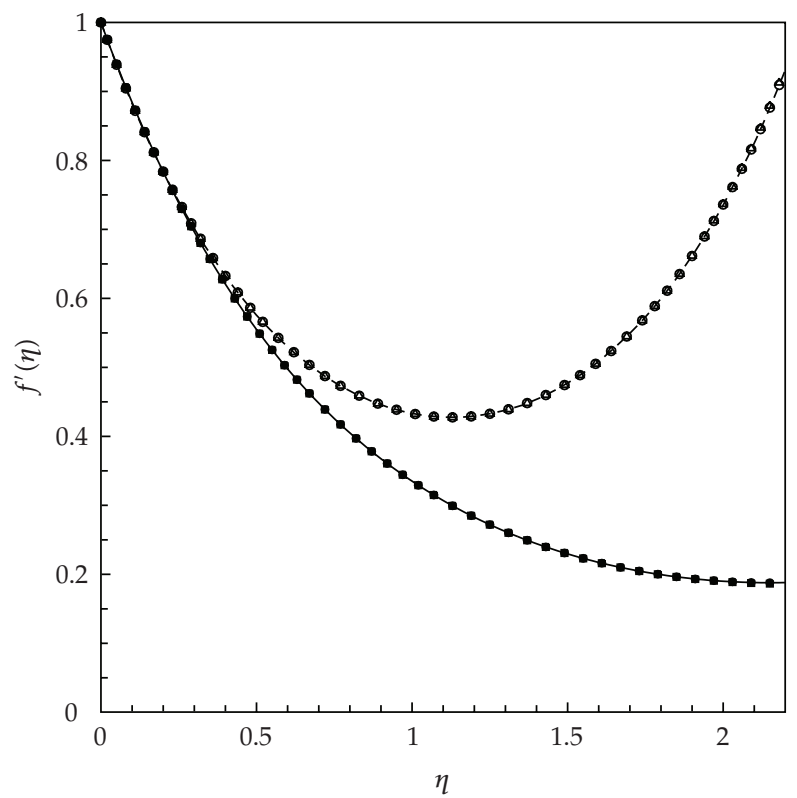

- Numerical, $S=0.8$

$\quad$ DTM, $S=0.8$

- Numerical, $S=1.2$

- DTM-Padé $[10,10]$,

$\triangle \quad \mathrm{DTM}, S=1.2$ $S=0.8$

--- DTM-Padé $[10,10]$,

Figure 2: Comparison of the solutions obtained by the DTM and the DTM-Pade for $M n=0, E c=0$. 


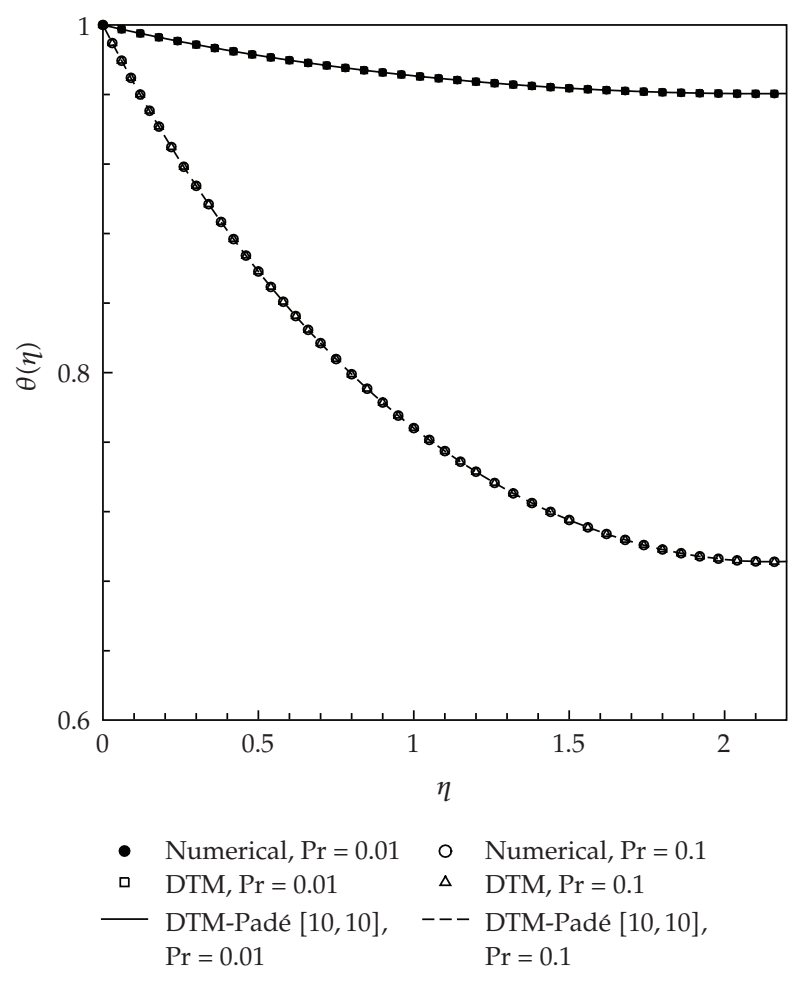

Figure 3: Comparison of the solutions obtained by the DTM and the DTM-Pade with numerical solutions for $M n=0, E c=0, S=0.8, \beta=2.151990$.

Fisher and Burger-Fisher equations. The Padé approximants [38] that often show superior performance over series approximations provide a successful tool and promising scheme for identical applications. For analytical solution, the convergence analysis was performed, and in (3.3), the $i$ value is selected equal to 20. The order of Padé approximation [L,M], $[10,10]$ has sufficient accuracy; on the other hand, if the order of Padé approximation increases, the accuracy of the solution increases but sometimes increasing the order of the DTMPadé significantly increases the volume of computations. The detail of this method is well addressed in [39].

Figure 1 presents the analytical and numerical solutions of dimensionless stream function $f(t)$ for $M n=0, E_{c}=0$, and two different values of unsteadiness parameter $S=0.8, \beta=2.151990$ and $S=1.2, \beta=1.127780$, respectively. It observed that $f(t)$ increases by increasing similarity variable $\eta$. Figure 2 indicates that increasing values of $\eta$ decreases the velocity profiles $f^{\prime}(\eta)$. Figures 3, 4, 5, and 6 display the variations of dimensionless temperature $\theta(\eta)$ for different values of prandtl number Pr and two different values of $S$. Comparison of the solutions obtained by the DTM and the DTM-Pade revealed that DTMPadé results are in excellent agreement with that of numerical solutions [36]. In fact, using the DTM-Padé aids to convergence of the DTM impressively.

\section{Conclusion}

In the present study, the DTM and DTM-Padé were used to find analytical solutions of magnetohydrodynamics boundary-layer equations. It was found that DTM-Padé is a 


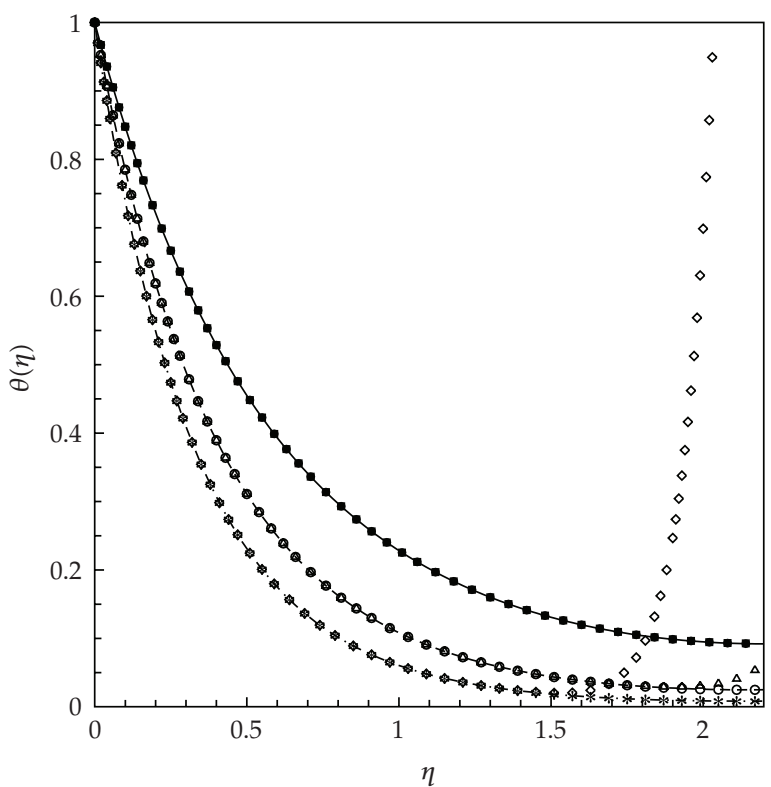

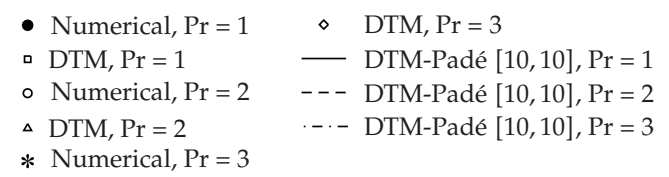

Figure 4: Comparison of the solutions obtained by the DTM and the DTM-Pade with numerical solutions for $M n=0, E c=0, S=0.8, \beta=2.151990$.

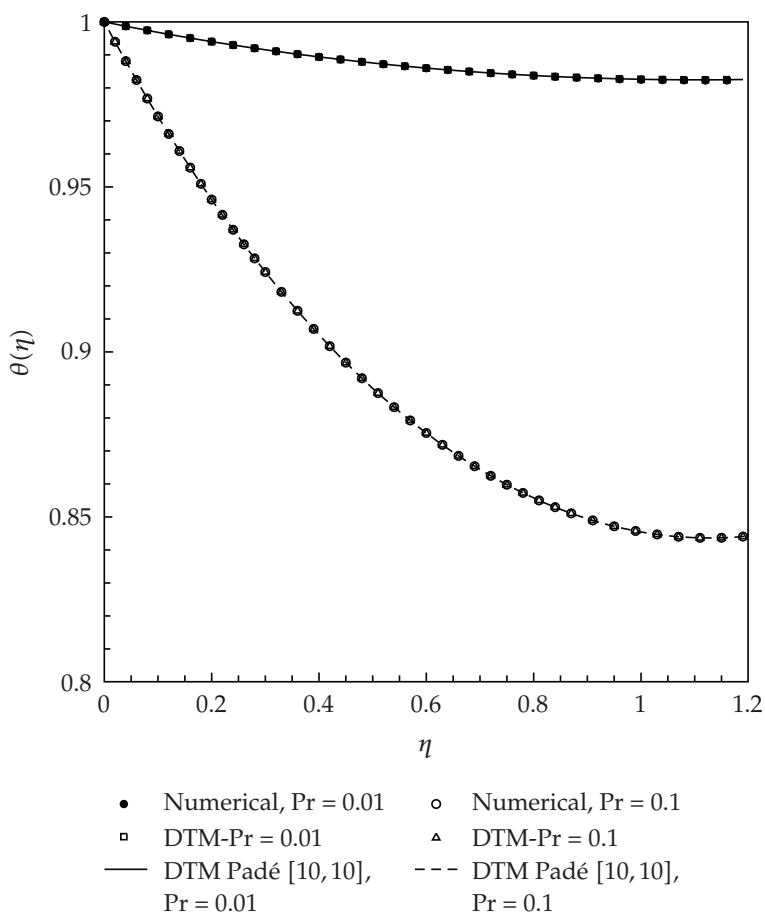

Figure 5: Comparison of the solutions obtained by the DTM and the DTM-Pade with numerical solutions for $M n=0, E c=0, S=1.2, \beta=1.127780$. 


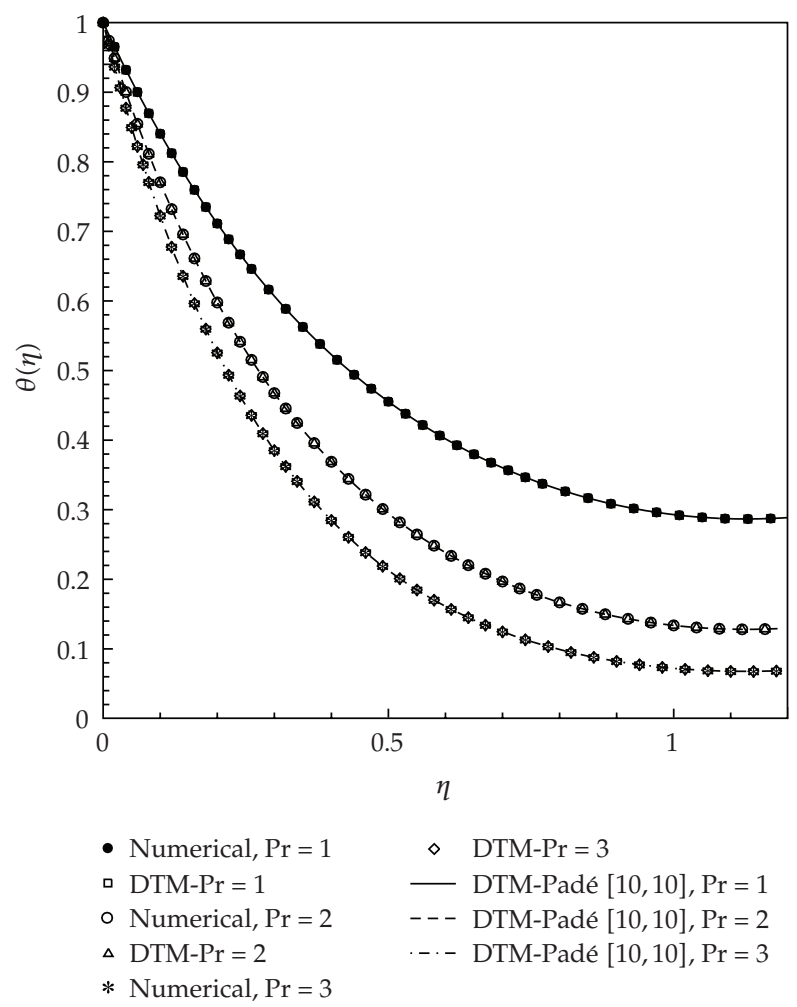

Figure 6: Comparison of the solutions obtained by the DTM and the DTM-Pade with numerical solutions for $M n=0, E c=0, S=1.2, \beta=1.127780$.

powerful method for solving problems consisting of systems of nonlinear differential equations. In this paper, a reliable algorithm is presented based on the DTM and DTMPadé to solve some nonlinear equations. Comparison between the solutions obtained by the DTM and DTM-Padé with numerical solution (fourth-order Runge-Kutta) remarked that the accuracy of DTM-Padé is very good. The method has been applied directly without requiring linearization, discretization, or perturbation. The obtained results certify the reliability of the algorithm and give it a wider applicability to nonlinear differential equations.

\title{
Nomenclature
}

\author{
$b$ : Stretching rate $\left[\mathrm{s}^{-1}\right]$ \\ $U$ : Sheet velocity $\left[\mathrm{m} \mathrm{s}^{-1}\right]$ \\ $x$ : Horizontal coordinate $[\mathrm{m}]$ \\ $y$ : Vertical coordinate [m] \\ $u$ : Horizontal velocity component $\left[\mathrm{m} \mathrm{s}^{-1}\right]$ \\ $v$ : Vertical velocity component $\left[\mathrm{m} \mathrm{s}^{-1}\right]$ \\ $T$ : Temperature [K] \\ $t: \quad$ Time [s] \\ $h$ : Film thickness [m] \\ $S$ : Unsteadiness parameter, $\alpha / b$ \\ $C_{p}$ : Specific heat $\left[\mathrm{J} \mathrm{kg}^{-1} \mathrm{~K}^{-1}\right]$
}


$f: \quad$ Dimensionless stream function, equation(2.10)

Pr: Prandtl number, $v / k$

Ec: $\quad$ Eckert number, $U^{2} / C_{p}\left(T_{s}-T_{0}\right)$

$M n$ : Magnetic parameter, $\sigma B_{0}^{2} / \rho b$

$q: \quad$ Heat flux, $-k(\partial T / \partial y)\left[\mathrm{J}\left(\mathrm{s}^{-1}\right) \mathrm{m}^{-2}\right]$

$\mathrm{Re}_{x}$ : Local Reynolds number, $U x / v$

$N u_{x}$ : Local Nusselt number, equation (2.23).

\section{Greek symbols}

$\alpha$ : Constant $\left[\mathrm{s}^{-1}\right]$

$\beta$ : Dimensionless film thickness

$\eta$ : Similarity variable, equation(2.12)

$\theta$ : Dimensionless temperature, equation(2.11)

$k$ : Thermal diffusivity $\left[\mathrm{m}^{2} \mathrm{~s}^{-1}\right]$

$\mu$ : Dynamic viscosity $\left[\mathrm{kg} \mathrm{m}^{-1} \mathrm{~s}^{-1}\right]$

$v$ : Kinematic viscosity $\left[\mathrm{m}^{2} \mathrm{~s}^{-1}\right]$

$\rho$ : Density $\left[\mathrm{kg} \mathrm{m}^{-3}\right]$

$\tau$ : Shear stress, $\mu \partial u / \partial y\left[\mathrm{kgm}^{-1} \mathrm{~s}^{-2}\right]$

$\psi$ : Stream function $\left[\mathrm{m}^{2} \mathrm{~s}^{-1}\right]$.

\section{Subscripts}

$o$ : Origin

ref: Reference value

s: Sheet

$x$ : Local value.

\section{Superscripts}

': First derivative

$"$ Second derivative

"': Third derivative.

\section{Acknowledgment}

The authors thank anonymous reviewers for their valuable comments.

\section{References}

[1] A. M. Lyapunov, The General Problem of the Stability of Motion, Taylor \& Francis, London, UK, 1992, English translation.

[2] J.-H. He, "Homotopy perturbation technique," Computer Methods in Applied Mechanics and Engineering, vol. 178 , no. 3-4, pp. 257-262, 1999.

[3] M. Dehghan and F. Shakeri, "Solution of an integro-differential equation arising in oscillating magnetic fields using He's homotopy perturbation method," Progress in Electromagnetic Research, vol. 78, pp. 361-376, 2008. 
[4] M. Dehghan and F. Shakeri, "Use of He's homotpy perturbation method for solving a partial differential equation arising in modeling of flow in porous media," Journal of Porous Media, vol. 11, pp. 765-778, 2008.

[5] M. Dehghan and F. Shakeri, "The numerical solution of the second Painlevé equation," Numerical Methods for Partial Differential Equations, vol. 25, no. 5, pp. 1238-1259, 2009.

[6] M. Dehghan and J. Manafian, "The solution of the variable coefficients fourth-order parabolic partial differential equations by the homotopy perturbation method," Zeitschrift für Naturforschung A, vol. 64a, pp. 411-419, 2009.

[7] F. Soltanian, M. Dehghan, and S. M. Karbassi, "Solution of the differential-algebraic equations via homotopy perturbation method and their engineering applications," International Journal of Computer Mathematics. In press (2009) DOI 10.1080.

[8] A. Saadatmandi, M. Dehghan, and A. Eftekhari, "Application of He's homotopy perturbation method for non-linear system of second-order boundary value problems," Nonlinear Analysis: Real World Applications, vol. 10, no. 3, pp. 1912-1922, 2009.

[9] F. Shakeri and M. Dehghan, "Inverse problem of diffusion equation by He's homotopy perturbation method," Physica Scripta, vol. 75, pp. 551-556, 2007.

[10] M. Dehghan and R. Salehi, "A semi-numeric approach for solution of the Eikonal partial differential equation and its applications," Numerical Methods for Partial Differential Equations, vol. 26, pp. 702-722, 2009.

[11] M. Dehghan and F. Shakeri, "Solution of a partial differential equation subject to temperature overspecification by He's homotopy perturbation method," Physica Scripta, vol. 75, no. 6, pp. 778787, 2007.

[12] A. V. Karmishin, A. I. Zhukov, and V. G. Kolosov, Methods of Dynamics Calculation and Testing for ThinWalled Structures, Mashinostroyenie, Moscow, Russia, 1990.

[13] M. Dehghan, M. Shakourifar, and A. Hamidi, "The solution of linear and nonlinear systems of Volterra functional equations using Adomian-Pade technique," Chaos, Solitons and Fractals, vol. 39, no. 5, pp. 2509-2521, 2009.

[14] M. Dehghan and F. Shakeri, "The use of the decomposition procedure of Adomian for solving a delay differential equation arising in electrodynamics," Physica Scripta, vol. 78, Article ID 065004, 11 pages, 2008.

[15] J. H. He, "A new approach to non-linear partial differential equations," Communications in Nonlinear Science and Numerical Simulation, vol. 2, no. 4, 1997.

[16] M. M. Rashidi and H. Shahmohamadi, "Analytical solution of three-dimensional Navier-Stokes equations for the flow near an infinite rotating disk," Communications in Nonlinear Science and Numerical Simulation, vol. 14, no. 7, pp. 2999-3006, 2009.

[17] T. Hayat, C. Fetecau, and M. Sajid, "Analytic solution for MHD transient rotating flow of a second grade fluid in a porous space," Nonlinear Analysis: Real World Applications, vol. 9, no. 4, pp. 1619-1627, 2008.

[18] T. Hayat, T. Javed, and M. Sajid, "Analytic solution for MHD rotating flow of a second grade fluid over a shrinking surface," Physics Letters A, vol. 372, no. 18, pp. 3264-3273, 2008.

[19] M. M. Abdelkhalek, "Heat and mass transfer in MHD flow by perturbation technique," Computational Materials Science, vol. 43, pp. 384-391, 2008.

[20] M. S. Abel and M. M. Nandeppanavar, "Heat transfer in MHD viscoelastic boundary layer flow over a stretching sheet with non-uniform heat source/sink," Communications in Nonlinear Science and Numerical Simulation, vol. 14, no. 5, pp. 2120-2131, 2009.

[21] A. Ishak, R. Nazar, and I. Pop, "MHD boundary-layer flow of a micropolar fluid past a wedge with constant wall heat flux," Communications in Nonlinear Science and Numerical Simulation, vol. 14, no. 1, pp. 109-118, 2009.

[22] K. V. Prasad, D. Pal, and P. S. Datti, "MHD power-law fluid flow and heat transfer over a nonisothermal stretching sheet," Communications in Nonlinear Science and Numerical Simulation, vol. 14, pp. 2178-2189, 2009.

[23] C. Y. Wang, "Analysis of viscous flow due to a stretching sheet with surface slip and suction," Nonlinear Analysis: Real World Applications, vol. 10, no. 1, pp. 375-380, 2009.

[24] C. Y. Wang, "Exact solutions of the steady-state Navier-Stokes equations," Annual Review of Fluid Mechanics, vol. 23, pp. 159-177, 1991. 
[25] J. K. Zhou, Differential Transformation and Its Applications for Electrical Circuits, Huazhong University Press, Wuhan, China, 1986.

[26] C. K. Chen and S. H. Ho, "Solving partial differential equations by two-dimensional differential transform method," Applied Mathematics and Computation, vol. 106, no. 2-3, pp. 171-179, 1999.

[27] M.-J. Jang, C.-L. Chen, and Y.-C. Liu, "Two-dimensional differential transform for partial differential equations," Applied Mathematics and Computation, vol. 121, no. 2-3, pp. 261-270, 2001.

[28] I. H. A.-H. Hassan, "Different applications for the differential transformation in the differential equations," Applied Mathematics and Computation, vol. 129, no. 2-3, pp. 183-201, 2002.

[29] F. Ayaz, "On the two-dimensional differential transform method," Applied Mathematics and Computation, vol. 143, no. 2-3, pp. 361-374, 2003.

[30] F. Ayaz, "Solutions of the system of differential equations by differential transform method," Applied Mathematics and Computation, vol. 147, no. 2, pp. 547-567, 2004.

[31] A. Kurnaz, G. Oturanç, and M. E. Kiris, "n-dimensional differential transformation method for solving PDEs," International Journal of Computer Mathematics, vol. 82, no. 3, pp. 369-380, 2005.

[32] I. H. A.-H. Hassan, "Comparison differential transformation technique with Adomian decomposition method for linear and nonlinear initial value problems," Chaos, Solitons and Fractals, vol. 36, no. 1, pp. 53-65, 2008.

[33] F. Kangalgil and F. Ayaz, "Solitary wave solutions for the KdV and $\mathrm{mKdV}$ equations by differential transform method," Chaos, Solitons and Fractals, vol. 41, no. 1, pp. 464-472, 2009.

[34] M. Subhas Abel and N. Mahesha, "Heat transfer in a liquid film over an unsteady stretching surface with viscous dissipation in presence of external magnetic field," Applied Mathematical Modelling, vol. 33, pp. 3430-3441, 2009.

[35] I. H. Abdel-Halim Hassan, "Comparison differential transformation technique with Adomian decomposition method for linear and nonlinear initial value problems," Chaos, Solitons and Fractals, vol. 36, no. 1, pp. 53-65, 2008.

[36] M. M. Rashidi and E. Erfani, "New analytical method for solving Burgers' and nonlinear heat transfer equations and comparison with HAM," Computer Physics Communications, vol. 180, pp. 1539-1544, 2009.

[37] H. N. A. Ismail and A. A. Abd Rabboh, "A restrictive Padé approximation for the solution of the generalized Fisher and Burger-Fisher equations," Applied Mathematics and Computation, vol. 154, no. 1, pp. 203-210, 2004.

[38] A.-M. Wazwaz, "Analytical approximations and Padé approximants for Volterra's population model," Applied Mathematics and Computation, vol. 100, no. 1, pp. 13-25, 1999.

[39] M. M. Rashidi, "The modified differential transform method for solving MHD boundary-layer equations," Computer Physics Communications, vol. 180, pp. 2210-2217, 2009. 


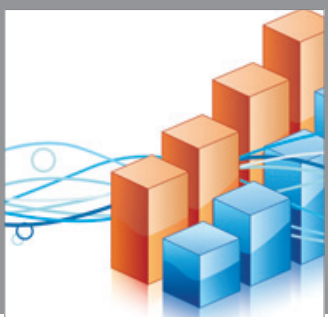

Advances in

Operations Research

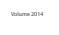

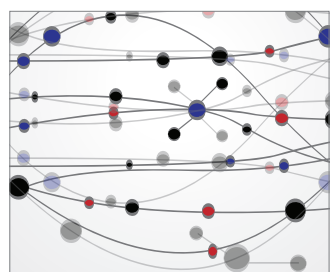

\section{The Scientific} World Journal
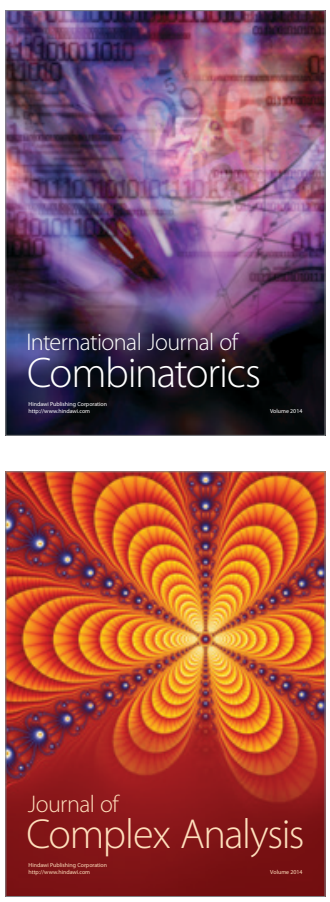

International Journal of

Mathematics and

Mathematical

Sciences
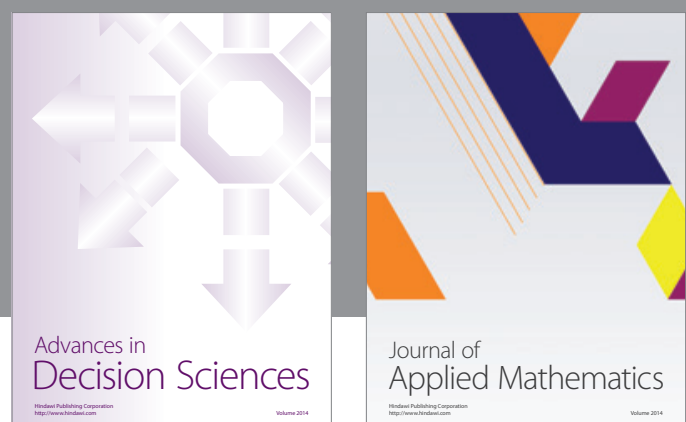

Journal of

Applied Mathematics
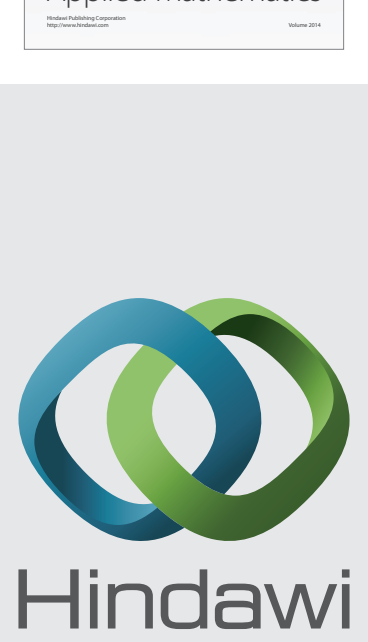

Submit your manuscripts at http://www.hindawi.com
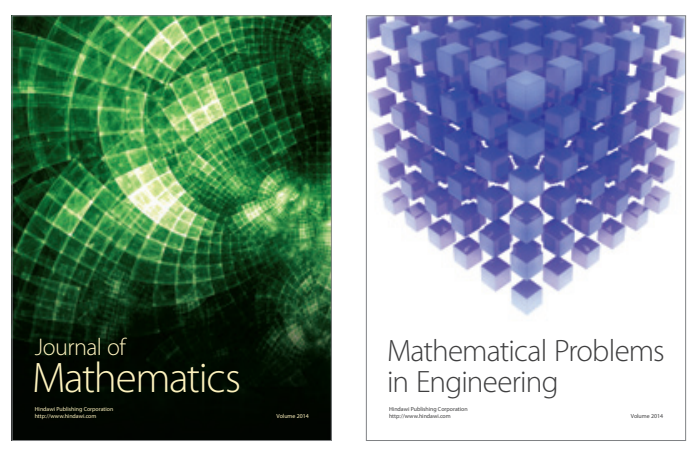

Mathematical Problems in Engineering
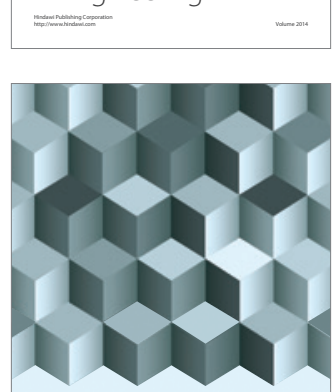

Journal of

Function Spaces
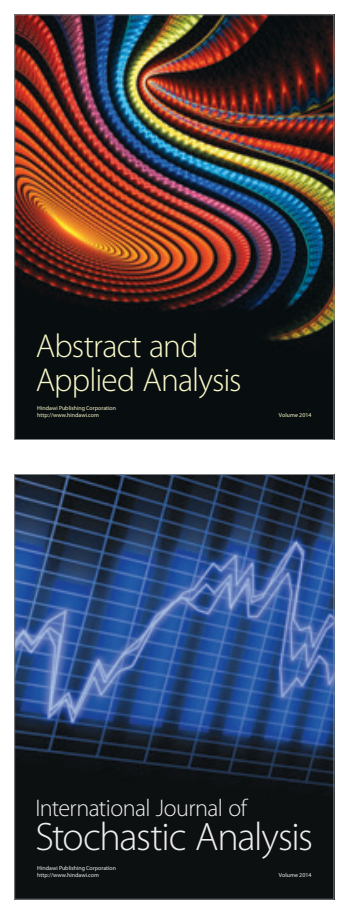

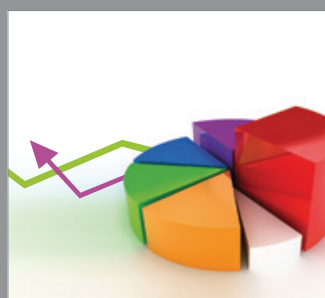

ournal of

Probability and Statistics

Promensencen
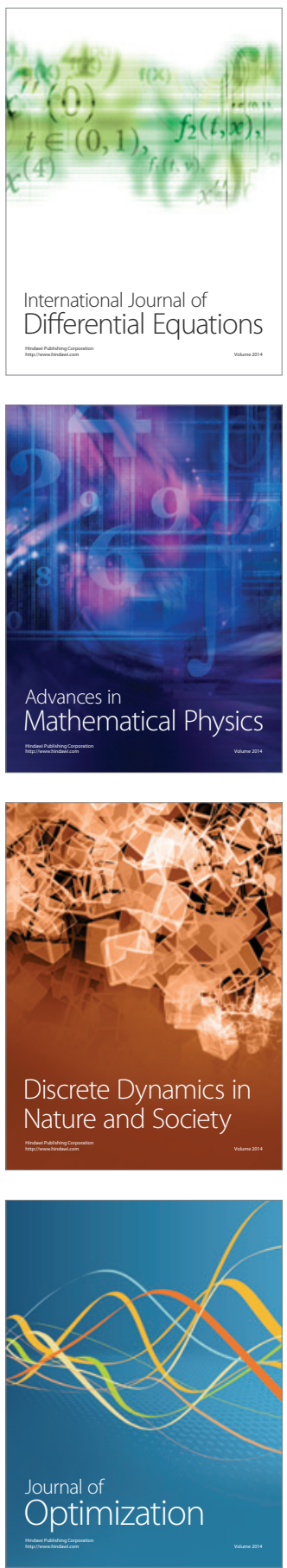